\title{
Retarding field analyzer for the Wendelstein 7-X boundary plasma
}

\author{
M. Henkel ${ }^{1}$, Y. Li ${ }^{\star 1,2}$, Y. Liang ${ }^{\star 1}$, P. Drews ${ }^{1}$, A. Knieps ${ }^{1}$, C. Killer ${ }^{3}$, D. Nicolai ${ }^{1}$, D. \\ Höschen $^{1}$, J. Geiger ${ }^{3}$, C. Xiao ${ }^{2,4}$, N. Sandri ${ }^{1}$, G. Satheeswaran ${ }^{1}$, S. Liu², \\ O.Grulke ${ }^{3}$, M. Jakubowski ${ }^{3}$, S. Brezinsek ${ }^{1}$, M. Otte ${ }^{3}$, O. Neubauer ${ }^{1}$, B. Schweer ${ }^{1}$, \\ G. $\mathrm{Xu}^{2}, \mathrm{~J} . \mathrm{Cai}^{2}$, and the W7-X team ${ }^{3}$ \\ ${ }^{1}$ Forschungszentrum Jülich GmbH, Institut für Energie- und Klimaforschung - Plasmaphysik, Partner of the Trilateral \\ Euregio Cluster (TEC), 52425 Jülich, Germany \\ ${ }^{2}$ Institute of Plasma Physics, Chinese Academy of Sciences, Hefei 230031, China \\ ${ }^{3}$ Max-Planck-Institut für Plasmaphysik, Greifswald, Germany \\ ${ }^{4}$ Department of Physics and Engineering Physics, University of Saskatchewan, 116 Science Place, Saskatoon, SK S7N \\ 5E2, Canada \\ *Contact email: y.li@fz-juelich.de and y.liang@fz-juelich.de
}

The ion temperature $\mathrm{T}_{\mathrm{i}}$ at the Wendelstein 7-X (W7-X) stellarator plasma boundary has been successfully measured by using a bi-directional multi-channel retarding field analyzer (RFA) probe. The RFA hardware and its upgrade for the two W7-X island divertor campaigns are presented, including the electronics. In this paper the experiences operating an RFA at W7-X are discussed, as well as the data analysis using a maximum coefficient of determination method to obtain the ion temperature based on the measured modulated ion current. Edge ion temperature profiles have been measured in the standard and high iota configurations.

\section{INTRODUCTION}

Edge ion temperatures $\left(\mathrm{T}_{\mathrm{i}}\right)$ in magnetic fusion test devices are rarely measured but yet are important for understanding edge plasma physics, especially the thermal equipartition. Currently, routinely operated spectroscopic observations cannot directly measure the fuel ion $\mathrm{T}_{\mathrm{i}}$ since the hydrogenic ions do not emit photons. And the low temporal and spatial resolution also limit its function. The alternative Ion Sensitive Probe (ISP) technique is in principle able to measure ion temperatures but suffers from space charge limitation problems in practical applications. ${ }^{1}$ The Retarding Field Analyzer (RFA) remains a practical tool to measure the edge ion temperature. To date, RFAs have been used on JET ${ }^{2,3}$, Tore Supra ${ }^{4}$, Alcator C-mod ${ }^{5}$, ISTTOK $^{6}$, STOR-M ${ }^{7}$, and EAST ${ }^{8}$ tokamaks and other plasma devices to measure the edge ion temperature profile. In this paper, we present the RFA operation in the plasma boundary of the Wendelstein 7-X (W7-X) device. W7-X is an optimized stellarator with great flexible magnetic configurations, including different edge island configurations with the island mode number $\mathrm{n} / \mathrm{m}=5 / 4,5 / 5,5 / 6 .{ }^{9,10}$ Generally, the RFA consists of one entrance slit to repel the thermal electrons, two or three grids to retard low energy ions and to prevent secondary electron emission, and one collector to measure the ion flux through the grids. The probe could measure the distribution of the charged particle velocity along the direction parallel to the magnetic field, $f\left(v_{\|}\right)$, 
based on the dependence of the collector current on the repelling voltage applied to the grid, or the commonly termed I-V characteristics. The I-V curve is then fitted to an exponential function to extract the ion temperature if a Maxwellian velocity distribution is assumed.

This paper focuses on the W7-X bi-directional RFA hardware and operation, and presents measured data process. Section II describes the design of the RFA probe. In Section III the measurements and their interpretation using a maximum coefficient of determination method are presented. Section IV summarizes the paper.

\section{RFA for W7-X}

The optimized stellarator W7-X aims to demonstrate quasi-steady state operation with plasma parameters, including ion temperatures, close to those of a future fusion power plant. It has a plasma volume $\sim 30 \mathrm{~m}^{3}$, major radius $\sim 5.5 \mathrm{~m}$ and effective minor radius $\sim 0.55 \mathrm{~m}$. The edge plasma parameters, plasma density and temperature near the separatrix $\mathrm{n}_{\mathrm{es}}$ and $\mathrm{T}_{\mathrm{es}}$, are roughly $\mathrm{n}_{\mathrm{es}}<3 \times 10^{19} \mathrm{~m}^{-3}, \mathrm{~T}_{\mathrm{es}}<100 \mathrm{eV}$ during the experimental operation ${ }^{11}$. The corresponding high power fluxes of the W7-X boundary plasma necessitate the RFA to be mounted onto a multiple-purpose manipulator ${ }^{12,13}$ (MPM) shaft to be first slowly moved to the parking position and then quickly inserted into the plasma with a maximum plunge distance of $35 \mathrm{~cm}$, a maximum acceleration of $30 \mathrm{~m} \mathrm{~s}^{-2}$, and a maximum speed of $2.5 \mathrm{~m} \mathrm{~s}^{-1}$. The MPM is located in the outer mid-plane with $Z=-171 \mathrm{~mm}$ and toroidal angle $\varphi=-159.26^{\circ}$, in between the characteristic bean shaped and triangular cross section. A variety of diagnostic probes ${ }^{14-16}$ mounted on the MPM have been developed for the measurements of edge profiles like the edge electron temperature and density, and the plasma flow. To measure the edge ion temperature, an RFA prototype has been first tested on EAST. ${ }^{17}$ Then RFA measurements were performed in the two first divertor operation campaigns of W7-X in 2017 and 2018. Here, we focus on the experiments of the second campaign in 2018 where both the RFA probe mechanical structure and electronics have been upgraded after several problems were encountered in the first (2017) campaign. These problems include damaged grids and wires, and anomalous -190V DC voltage on the graphite cover. These issues will be discussed in detail in sub-section C.

\section{A. RFA body design}

Figure 1 shows the design of a bi-directional 3-channel-array RFA head used for the W7-X. To follow the magnetic geometry of W7-X, the RFA head has been rotated 18 degrees upwards in the poloidal direction. The probe body consists of two identical analyzers mounted back to back. As shown in Fig. 1, the analyzer on each side contains a 3-mm thick tungsten entrance orifice plate (white color sheet), a newly installed thin tungsten entrance slit plate with a thickness of $50 \mu \mathrm{m}$, three successive grids (green color sheets), and three collector plates (white, blue and purple color sheets). The physical and electrical 
separation between the adjacent grid sheets is maintained by a ceramic spacer plate (orange color sheets) with a thickness of 1 mm.

Instead of the old sheet with the vertical slit array with a spacer width $~ 100 \mu \mathrm{m}$ in the W7-X 2017 campaign, the thin entrance slit plate (thickness $\sim 50 \mu \mathrm{m}$ ) has three, slits openings with a width $\sim 30 \mu \mathrm{m}$ and spacing between the slits is $4 \mathrm{~mm}$. The compact design allows $\mathrm{T}_{\mathrm{i}}$ measurement at three radial locations simultaneously. The slit width is allowed to repel the thermal electrons, which is required to be on the order of the Debye length $\left(\lambda_{D}=\sqrt{\varepsilon_{0} k_{B} T_{e} / n_{e} e^{2}}\right.$, where $\varepsilon_{0}$ the vacuum permittivity, $k_{B}$ the Boltzmann constant, $\mathrm{T}_{\mathrm{e}}$ electron temperature, $\mathrm{n}_{\mathrm{e}}$ the electron density, and e the elementary charge). $\lambda_{D}$ is in the range 5 $50 \mu \mathrm{m}$ in the W7-X scape-off-layer (SOL). The actual slit width of $\sim 25 \mu \mathrm{m}$ has been verified by a microscope (as shown in Fig. 2). To prevent high heat load plasma impinging on the thin entrance slit plate, the thick entrance orifices have been added at the front of the thin entrance plate. The front tungsten plate is cut with three trapezoid orifices. The angle between the lateral sides is $45^{\circ}$. The short base of the trapezoid slit with a width of $\sim 300 \mu \mathrm{m}$ is attached to the thin entrance slit. The thick plate is made by wire eroding and the thin entrance slits are cut by laser. Each grid is made out of a flat tungsten blank (thickness 50 $\mu \mathrm{m})$ on which a regular two dimensional array of square openings is machined. The sides of the square opening is $0.4 \mathrm{~mm}$ and the bar width between the opening is $0.1 \mathrm{~mm}$. The optical transmission of the grid is 64\%. In the 2018 campaign, the shape of the grid base has been optimized to minimize the surface area to decrease the capacitance between the grid and the collector. On the base of the grids and thin slit plate a small section of grid-style holes has been machined so the $0.3 \mathrm{~mm}$ diameter copper wires can be crimped to the section to transmit electrical signals.

After the bias voltage is applied to the grids, the ions would first pass through an electrical field changing in time and location inside the RFA cavity. If the ion energy were larger than the bias voltage $\mathrm{V}_{2}$ on Grid 2, ions would reach the collectors and contribute to the total current measured by the external electronic circuits. Each collector fixed at the ceramic base measures the ion current passing through the corresponding entrance slit. The bi-directional RFA components are fastened by two sets of screws and nuts which are electrically insulated with the six grids. The two thick entrance slit plates in both sides are electrically connected with each other and self-biased negatively with respect to the plasma space potential. A boron nitride cover (green color cover in Fig. 1) is chosen to protect the RFA components.

In addition to the RFA components described, at the RFA probe top, there are also five tungsten Langmuir tips and one stainless steel gas inlet (tube inner diameter $2.5 \mathrm{~mm}$ ). These Langmuir probes are $2 \mathrm{~mm}$ in diameter and 3mm long over the outer cover of the boron nitride cover. $0.5 \mathrm{~mm}$ diameter wires are used to connect with the Langmuir tips to the external circuitry. 
During the RFA experiment, three of the six channels have measured the ion current, demonstrating the soundness of the RFA hardware design and assembly. Only three of total five Langmuir tips have been used as a triple probe due to the limitation on the number feedthrough channels on the MPM system.

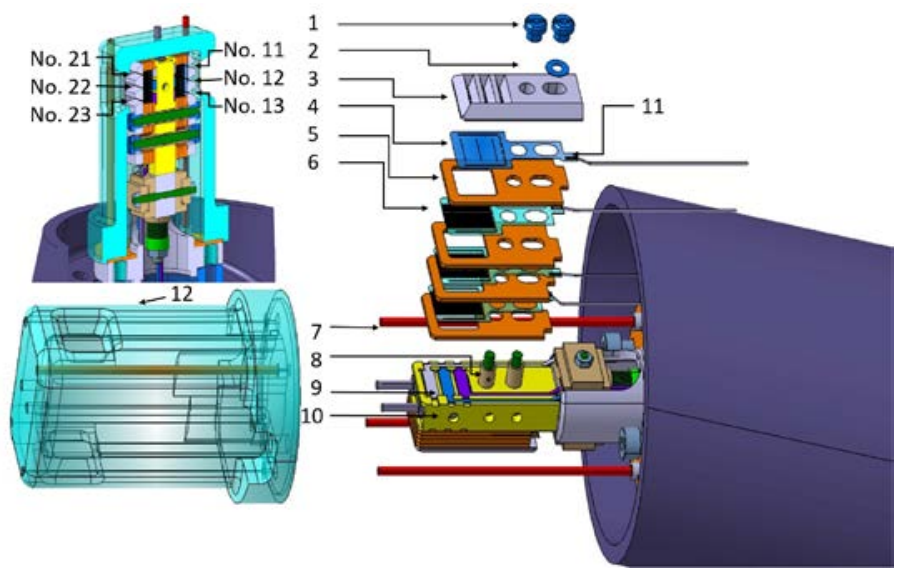

FIG. 1. Sectional and exploded isometric decomposition views of the W7-X multi-channel RFA probe head, which contains 1, screw; 2, gasket; 3, orifice plate; 4, thin entrance slit plate; 5, ceramic spacer plate; 6, grid; 7, Langmuir probe; 8, nut; 9, collector; 10, ceramic base; 11, grid-style holes; 12, boron nitride cover, and the channel number named as No. 11, 12, 13 in one side and No. 21, 22, and 23 in the other side.

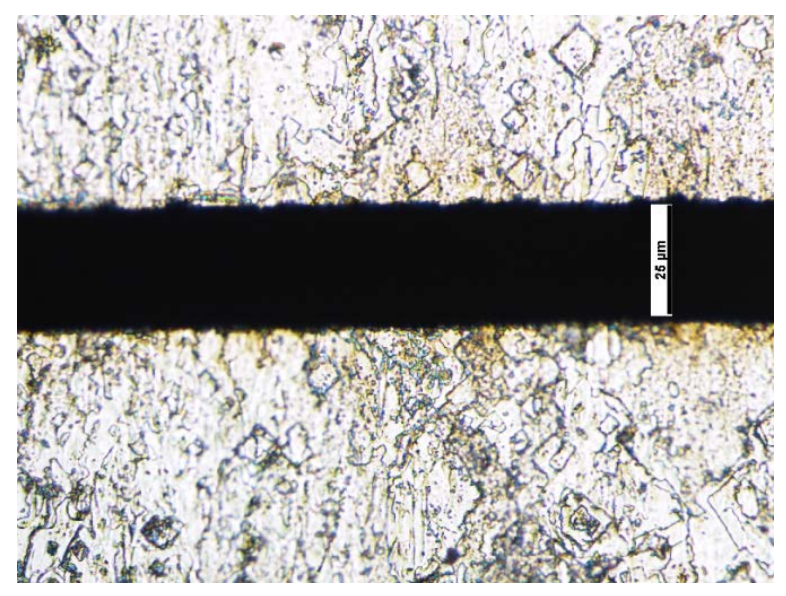

FIG. 2. Thin entrance slit width measured by a microscope

\section{B. RFA circuit design}

The RFA circuit has been designed to flexibly control and monitor the bias voltages on the entrance slit plates and the grids. An overview of the circuit schematic for RFA measurement is shown in Fig. 3. In this figure, most of the corresponding components are connected in parallel into the power supply. However, each of the six RFA channels uses a separate collector plate for independent ion current measurement. The circuit design allows measurement of ion current in a wide range from 
$1 \mathrm{~mA}$ to $10 \mathrm{~A}$ by remotely switching the current sampling shunt resistor in five steps from $100 \Omega$ to $10 \mathrm{~m} \Omega$. The bias voltage for the slit plates and six grids have been monitored at the points indicated by the red solid dots in Fig. 3 through a circuit with an input resistance of $1 \mathrm{M} \Omega$. The power supply parameters can be remotely programmed based on the experimental needs. Table 1 lists the biasing supply parameters including their types of waveforms, adjustable voltage range, and maximum current. Figure 3 also shows the triple probe circuit. The circuit ground is connected to the W7-X vessel ground. The current and voltage signals are measured via optoelectronic isolators by the data acquisition (DAQ) system.

During the RFA experiments, the RFA circuit has been operated with two floating entrance slits to protect the wires from possible transient high current. Grids 1 and 3 are biased by a negative DC voltage, -100 V and -200 V, respectively. Grid 2 is biased by a sweep voltage supply at a 1-kHz frequency.

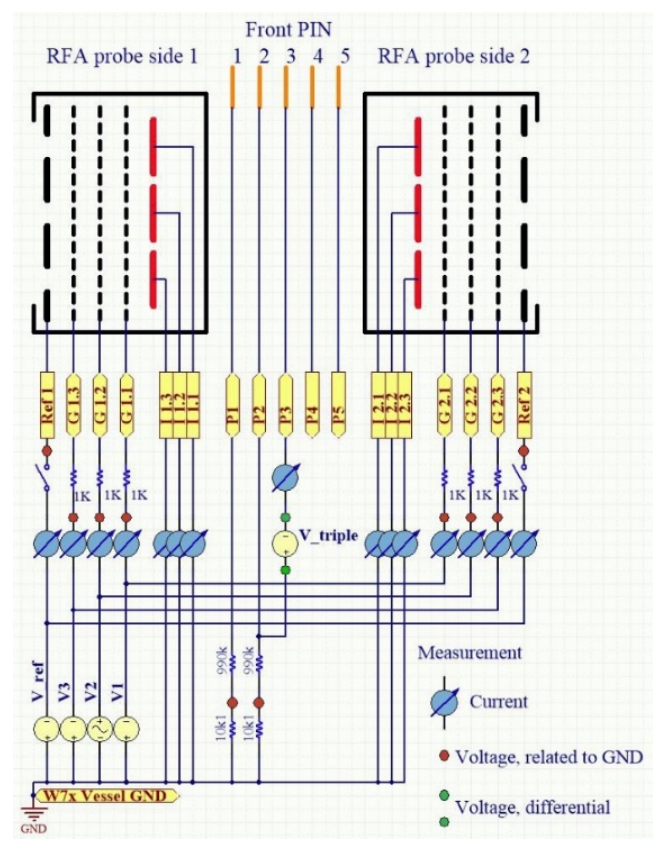

FIG. 3. The RFA circuit sketch, where Pins 1, 2 and 3 are used to measure the plasma floating potential, positive potential, and ion saturated current, respectively.

TABLE I. RFA electronic scheme.

\begin{tabular}{cccc}
\hline \hline System & Type & $\begin{array}{c}\text { Voltage } \\
\text { range }(V)\end{array}$ & $\begin{array}{c}\text { Max } \\
\text { current }(\mathrm{A})\end{array}$ \\
\hline $\mathrm{V}_{\text {ref }}$ & DC & $0-160$ & 10 \\
$\mathrm{~V}_{1}$ & DC & $0-320$ & 10 \\
$\mathrm{~V}_{2}$ & AC +DC & AC: \pm 300, & 2 \\
& offset & max $10 \mathrm{kHz}$ & 1 \\
& & DC: \pm 200 & 10 \\
$\mathrm{~V}_{3}$ & DC & $0-160$ & 10 \\
$\mathrm{~V}_{\text {triple }}$ & DC & $0-320$ & 10 \\
\hline \hline
\end{tabular}

\section{RFA operation in W7-X}


RFA has been performed in the two first divertor operation campaigns of W7-X in 2017 and 2018 and was encountered some issues in both the campaigns. Firstly, during the 2017 RFA experiment, the RFA grid sheets were destroyed as shown in Fig. 4. The wires connecting the grids were also broken. It was speculated that arcing may has occurred between the grids and destroyed the grid structure and wires. To solve this issues, a $1 \mathrm{k} \Omega$ high power resistor has been added to the six-grid circuits to reduce the current flowing through the grids. The carbon cover originally designed to be floating was anomalously biased with large -190V DC voltage which maybe caused by the broken wires. And then the carbon cover is replaced by a boron nitride cover. In addition, the RFA operation has been conducted much more carefully in the 2018 campaign. All the grids during the first plunge of RFA were without the bias voltage to clean the RFA cavity by hot plasma. And then the biased DC and AC powers were imposed to the grids step by step in the coming discharges.

Secondly, the plasma conditions of W7-X have been observed to affect the measurements of RFA adversely. Usually in $\mathrm{W} 7-\mathrm{X}$, the plasma potential in SOL is strongly positive in low plasma density regime ${ }^{18}$. Because the RFA entrance slit is floated, its voltage could exceed the sweeping voltage on Grid 2. The ion influx inside the RFA cavity then would not be modulated by the sweeping voltage. The collected current on the collect becomes saturated. A typical example is shown in Fig. 5. In this discharge \#180816021, the ECRH heating power is $2 \mathrm{MW}$ and the plasma density is nearly constant, $\sim 2.5 \times 10^{19} \mathrm{~m}^{-2}$, controlled by gas puffing. The magnetic configuration is EIM+252, meaning the standard configuration with a field strength of $2.52 \mathrm{~T}$ at phi $=0^{\circ}$ (ECRH-launching plane) at the magnetic axis. The floating potential measured by the entrance slit gradually increases as the probe moves to the separatrix. Larger voltage on the entrance slit leads to smaller modulating ion flux range. Care should be taken to compare the measured floating potentials from the Langmuir probes to the biasing applied the RFA grids to preselect faulty. To measure the modulated ion current in the current setup, the RFA experiment has to been limited in the high plasma density regime, i.e., $\mathrm{n}_{\mathrm{el}}>7 \times 10^{19} \mathrm{~m}^{-2}$.

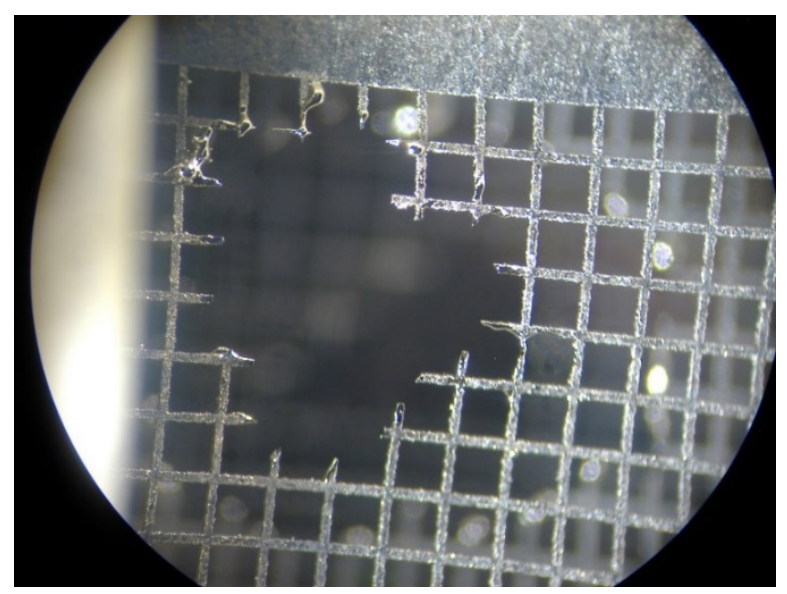

FIG. 4. Photo of the damaged RFA grids during the first island divertor campaign. 


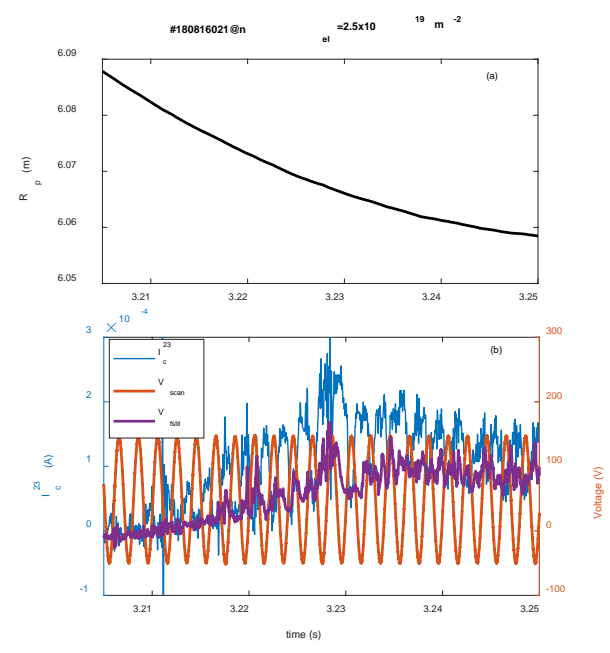

FIG. 5. Evolution of (a) major radial position and (b) modulated ion currents of channel 23, scan voltage, and floating potential on the entrance slit.

\section{Ion temperature measurement}

\section{A. Ion temperature interpretation}

The measured RFA current can be expressed by the following formula, assuming that the fuel ions of charge eZ $\mathrm{Z}_{\mathrm{i}}$ dominate the incident ion flux,

$$
I_{C}=A_{\text {beam }} e Z_{i} \int_{\sqrt{2 e Z\left(V_{2}-V_{s}\right) / m_{i}}}^{\infty} \xi_{\text {total }} v_{\|} f\left(v_{\|}\right) d v_{\|}
$$

where $I_{C}$ is the RFA collector current, $A_{\text {beam }}$ the ion beam area, $e$ the electron charge, $m_{i}$ the ion mass, $Z_{i}$ the ion charge number, $V_{2}$ the retarding sweep voltage, $V_{s}$ the sheath potential, $\xi_{\text {total }}$ the total ion transmission coefficient, $f\left(v_{\|}\right)$the ion distribution function for the velocity component parallel to the magnetic field line. Assuming that the ion velocity distribution function in the unperturbed plasma near the RFA probe is Maxwellian, Eq.1 can be simplified

$$
I_{C}= \begin{cases}I_{0}, & V_{2}<V_{s} \\ I_{0} e^{-\left(V_{2}-V_{s}\right) Z / T_{i}}, & V_{2} \geq V_{s}\end{cases}
$$

where $I_{0}$ is the maximum ion current when no ions are repelled by the retarding potential $V_{2}$.

During the RFA experiment, the modulated ion currents have been measured by three collector channels (channel numbers 11, 12 and 23) as the voltage applied to Grid 2 is scanned from -20V to 200V. Since the modulated signal is weak and submerged in noise including the plasma fluctuation, the auto-correlation power spectral densities were calculated to extract the modulated 
signals. The result is shown in Fig. 6, where the power spectral density for each channel has been calculated in the before (dash line) and after (solid line) entering plasma phases. In this discharge \#180905020, the main plasma is confined by the standard configuration and maintained by 3MW X2 heated electron cyclotron resonance heating (ECRH) power. The almost constant central line integrated electron density $n_{\mathrm{el}}=7 \times 10^{19} \mathrm{~m}^{-2}$ is controlled by gas puffing. The RFA was plunged into the plasma at 5.2s. Before the probe entering the plasma, these three channels can only measure the background noise and show similar noise spectrum with dominant noise frequency mainly larger than $80 \mathrm{kHz}$. After the probe inside the SOL and close to the separatrix, the plasma turbulence becomes prominent near frequency $\sim 200 \mathrm{kHz}$ and enhance larger fluctuation for higher frequency $(>10 \mathrm{kHz})$ in channels 11 and 12 than in channel 23 even that all these channels are designed to behave identical hardware unit. The reason for this phenomenon is still unclear. In this case, to obtain clear modulated currents for I-V curve fitting, the collector currents were first low-pass filtered with cutoff frequency $10 \mathrm{kHz}$ for channels 11 and 12, and $30 \mathrm{kHz}$ for channel 23. And then they were subtracted by the induced current due to the inductive coupling between Grid 2 and the collector. Figure 7 shows the waveforms of the collector currents and scan voltage as the probe moves over a distance of $\sim 1 \mathrm{~cm}$ into the plasma. After the smoothing process, a clear modulated current has been observed in channel 23. While the collector currents on channels 11 and 12 still contain a certain amount of noise which pollutes the valuable modulated current.

The ion temperature was obtained by fitting the $I_{c}-V_{2}$ curve based on Eq. (2) and assuming $Z=1$ (hydrogen plasma). Since the initial input setting could affect the fitting result, the initial parameters in Eq. (2) have been scanned, named as $T_{i}^{\text {scan }}$ and $V_{s}^{\text {scan }}$, to obtain the best fitting curve with maximum coefficient of determination $\mathrm{R}^{2}$, which is assumed to be closest to the plasma parameters. The output parameters of fitting curve, named as $T_{i}^{f i t}$ and $V_{s}^{f i t}$ would also change with different initial parameters. The results of $\mathrm{R}^{2}$ and the corresponding $T_{i}^{f i t}$ as functions of the scanned $T_{i}^{\text {scan }}$ and $V_{s}^{\text {scan }}$ are shown in Fig. 8(a), where the experimental data used for fitting is from channel 11 and within the time interval [5.246 5.248]s for two scan periods as shown in the rectangular shaded area of Fig. 7. The maximum $\mathrm{R}^{2}$ point is expressed by a red circle. As can be seen in this figure, $T_{i}^{f i t}$ gradually decreased from 80 to $60 \mathrm{eV}$ and kept almost constant as increasing the input parameters of $T_{i}^{\text {scan }}$ and $V_{s}^{\text {scan }}$. The fitting counts of $T_{i}^{f i t}$ and $V_{s}^{f i t}$ displayed as a histogram in Fig. 8(b) shows that the maximum $\mathrm{R}^{2}$ points (red circle) is located near the largest number of areas. This indicates that the maximum $\mathrm{R}^{2}$ points seems also the most probable point. After obtained the parameters of $T_{i}^{f i t}$ and $V_{s}^{f i t}$ with maximum $\mathrm{R}^{2}$, the $\mathrm{I}_{\mathrm{c}}-\mathrm{V}_{2}$ curve fitting of three channels are plotted in Fig. 9. The fitting curve parameters of $T_{i}$ and $V_{s}$ are also shown in this figure. Note that the measurement radial position is still $\sim 2$ $\mathrm{cm}$ away from the separatrix $\left(R_{\text {sep }} \approx 6.04 \mathrm{~m}\right)$. Since the $\mathrm{I}_{\mathrm{c}}$ signals of channels 11 and 12 have much larger noise comparing 
with channel 23 as shown in Fig. 7, the $\mathrm{I}_{\mathrm{c}}-\mathrm{V}_{2}$ data shows much clearer exponential trend for channel 23 than in channels 11 and 12. As a consequence, the fitting uncertainty is much larger in channels 11 and 12 than in channel 23.

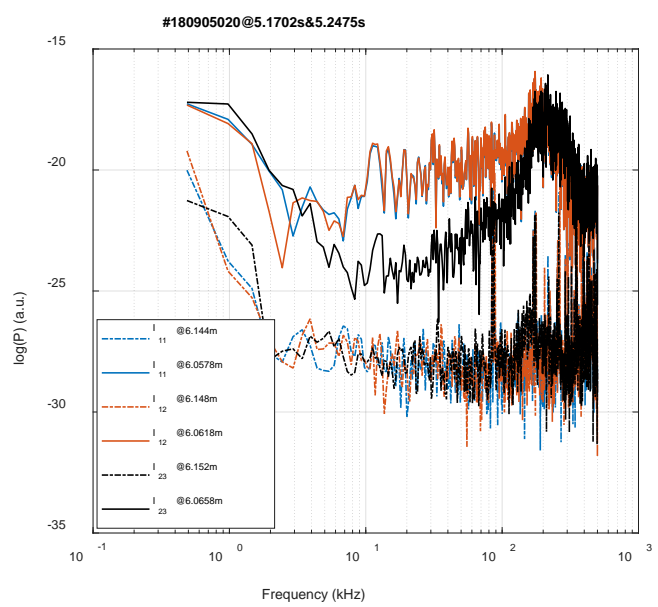

FIG. 6. The auto-correlation power spectral density of collector currents measured by channel 11, 12 and 23.

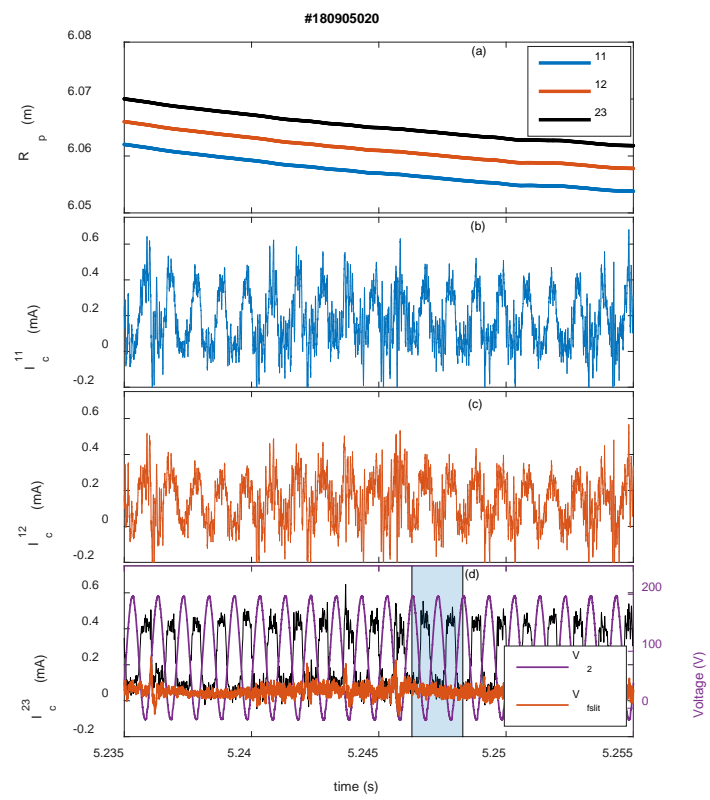

FIG. 7 Evolution of (a) major radial position and modulated ion currents of the channels 11 (b), 12 (c) and 23 (d). Note that the cutoff frequency are $10 \mathrm{kHz}$ in channel 11 and 12, and $30 \mathrm{kHz}$ in channel 23. 

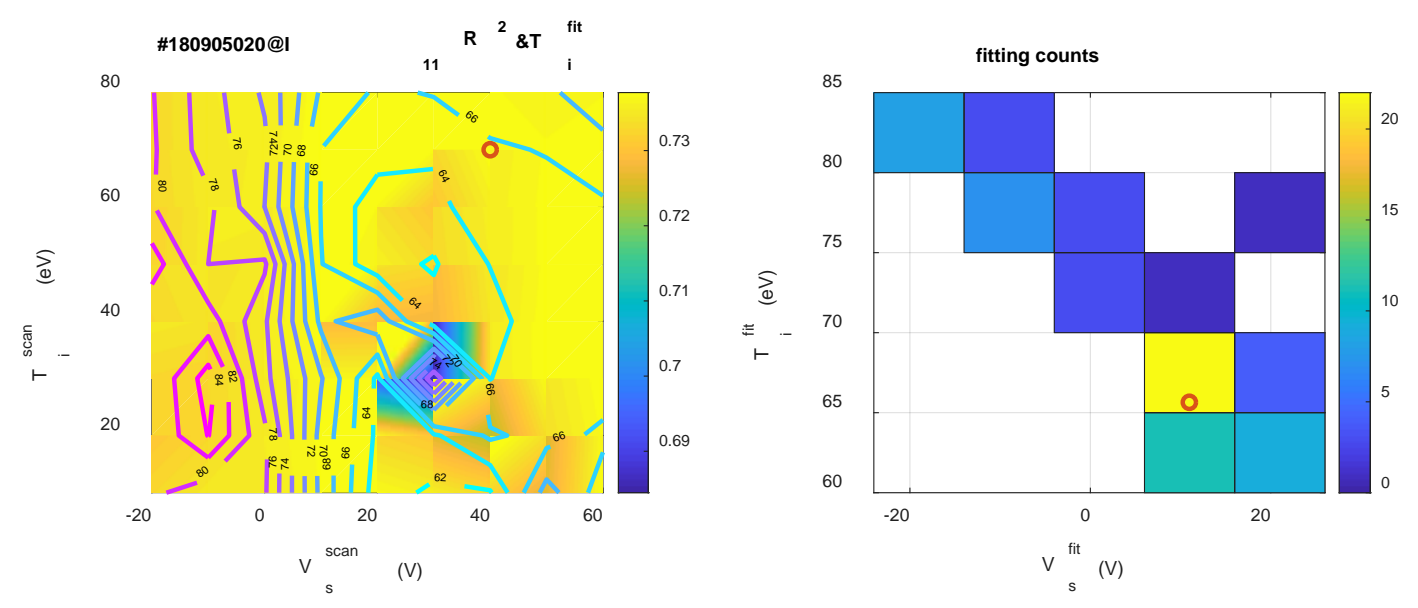

FIG. 8 The time interval corresponds to the shaded span as shown in Fig. 7. The maximum $\mathrm{R}^{2}$ is covered as red circle.

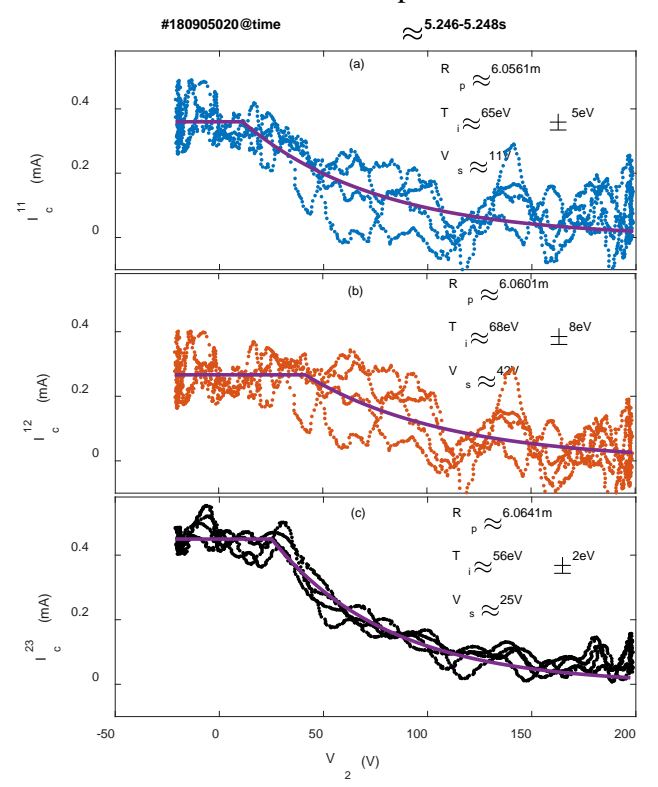

FIG. 9 I-V curve with two voltage scanning cycles and I-V curve fitting results. The time interval corresponds to the shaded span as shown in the lowest panel in Fig. 7.

\section{B. Ion temperature profile at the W7-X plasma boundary}

The retarding field analyzer was installed on the MPM during programs with the high iota and standard configuration in the 2018 campaign. In Fig. 10 the field line connection length distribution and Poincare map are shown, overlapped with the probe movement trace. In high iota configuration (FTM+252), the formed 5/4 edge island chain shifts inward both the separatrix and the SOL region compared with the standard configuration (EIM+252) which forms a 5/5 island chain in the plasma boundary. The major radius of the separatrix at the RFA movement $\mathrm{Z}$ position has been shifted inwards from $\mathrm{R}_{\text {sep }} \approx 6.04 \mathrm{~m}$, with the standard configuration, to $6.01 \mathrm{~m}$, with the high iota configuration. The radial width of the edge island in the high iota configuration is also much smaller than in the standard case. The fitted ion temperature profiles, in channels 11, 12 and 23 are shown in Fig. 11, where only the maximum coefficient of determination $\mathrm{R}^{2}$ larger than 0.7 is displayed. The connection length 
profile along the probe movement path are also covered into the figure. The plasma for FTM configuration is operated at 2 MW O2 heated ECRH power and $\mathrm{n}_{\mathrm{el}}=9 \times 10^{19} \mathrm{~m}^{-2}$. Since there are much lower heating power and higher plasma density, it's no wonder that the ion temperature is much lower in FTM than in EIM configuration. Because of the island configuration effect, the edge ion temperature profile has been observed to move further inwards when the configuration is changed from standard to high iota configuration. This profile shift is consistent with the connection length distribution. Besides, the ion temperature profile seems expanded and flattened by the larger island geometry in the standard configuration. The FLUX probe also measured that the plasma electron temperature and density profiles are much broader in standard configuration than in others ${ }^{11}$.

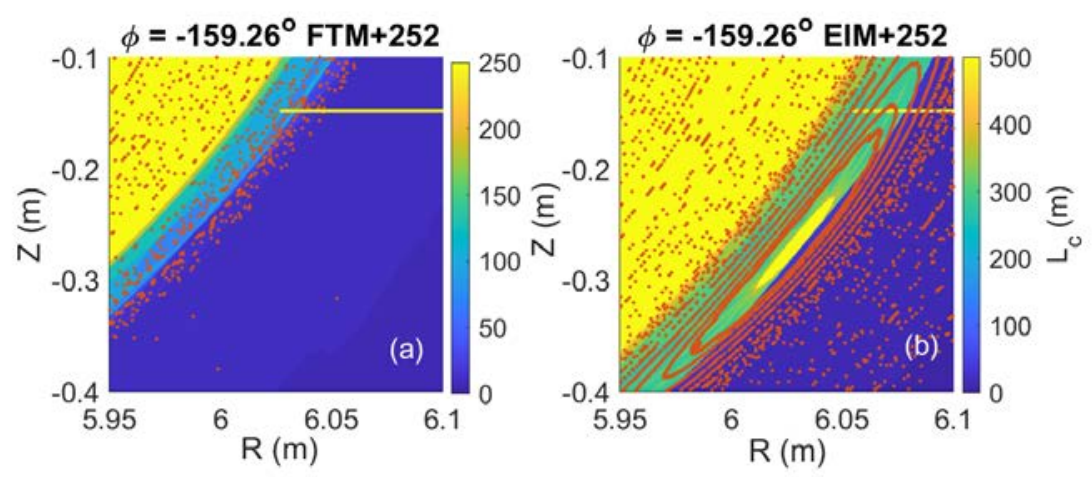

FIG. 10. Connection lengths profiles and superimposed magnetic configuration in (a) high iota configuration, FTM+252 and (b) standard configuration, EIM+252, where the color code is connection length, red dots are Poincare plot and yellow lines are MPM pathes.

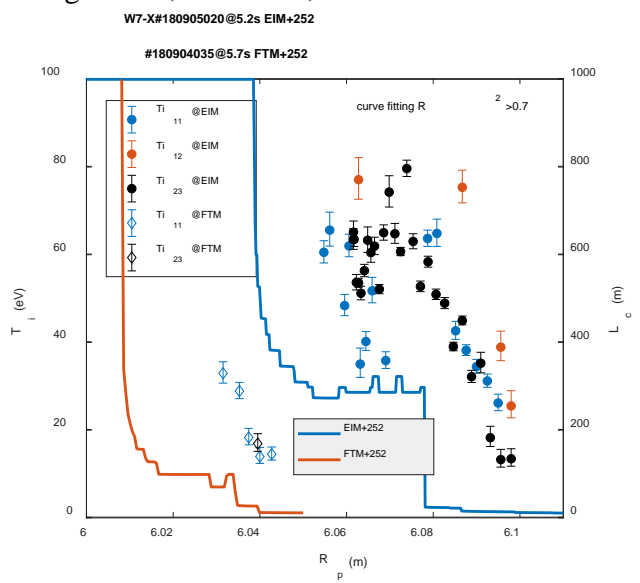

FIG. 11. Ion temperature profiles in high iota $(\mathrm{Rp}=6.032-6.043 \mathrm{~m})$ and standard configuration $(\mathrm{Rp}=6.055-6.1 \mathrm{~m})$ measured from channels 11, 12, and 23. The error bar of Ti represents the confidence interval of fit. The coefficient of determination, denoted $\mathrm{R}^{2}$, is $>0.7$.

\section{Summary}

The ion temperature, $\mathrm{T}_{\mathrm{i}}$, has been successfully measured in Wendelstein 7-X (W7-X) plasma boundary during the island divertor 2018 campaign using a bi-directional retarding field analyzer (RFA) probe array, which was previously commissioned at EAST and subsequently tested on W7-X during the first island divertor 2017 campaign. The RFA probe consists of in each 
side a front plate with 3 entrance orifices (width $300 \mu$ m, length $8 \mathrm{~mm}$ per orifice) on top of a newly added thin plate with 3 entrance slits (width $30 \mu \mathrm{m}$, length $8 \mathrm{~mm}$ per slit and thickness $50 \mu \mathrm{m}$ ) aligned and in electrical contact with the front plate, three successive grids, and three separate collector plates. The RFA circuit is designed to flexibly control and monitor the bias voltages on the entrance slit plates and the grids. Due to the difficulty in obtaining the $\mathrm{T}_{\mathrm{i}}$ in 2017 campaign, the RFA hardware has been upgraded with respect to both the probe mechanical structure and the electronics. The carbon cover of the probe was replaced by a boron nitride cover. Two Langmuir pins in the previous design have been extended to five pins on the top of the RFA probe to simultaneously measure the electron density, electron temperature and the turbulence information. The sampling circuits of all the grids were also upgraded to prevent the damage caused by arcing between the grids. To avoid large positive floating potential in the W7-X SOL, the probe has been limited to be operated in high density discharges $\left(\mathrm{n}_{\mathrm{el}}>7 \times 10^{19} \mathrm{~m}^{-2}\right)$. The ion temperature has been interpreted by utilizing a maximum coefficient of determination method. The edge ion temperature profile has been observed to be shifted inwards in high iota configuration comparing with the standard configuration.

\section{ACKNOWLEDGMENTS}

The authors deeply appreciate the continued research and operational efforts of the entire W7-X team. This work has been carried out within the framework of the EUROfusion Consortium, has received funding from the Euratom research and training programme 2014-2018 and 2019-2020 under grant agreement No 633053, and was supported by the International Postdoctoral Exchange Fellowship Program Nos. 20170009, the National Natural Science Foundation of China under Grant Nos. 11705237.

\section{REFERENCES}

${ }^{1}$ D. Brunner, B. LaBombard, R. Ochoukov, R. Sullivan and D. Whyte, Plasma Phys. Control. Fusion 55, 125004 (2013). ${ }^{2}$ H. Y. Guo, G. F. Matthews, S. J. Davies, S. K. Erents, L. D. Horton, R. D. Monk and P. C. Stangeby, Contrib. Plasma Phys. 36, 81 (1996).

${ }^{3}$ R. A. Pitts, R. Chavan, S. J. Davies, S. K. Erents, G. Kaveney, G. F. Matthews, G. Neill, J. E. Vince, I. Duran and J.-E. W. Contributor, Rev. Sci. Instrum. 74, 4644 (2003).

${ }^{4}$ M. Kočan, J. P. Gunn, M. Komm, J.-Y. Pascal, E. Gauthier and G. Bonhomme, Rev. Sci. Instrum. 79, 073502 (2008).

${ }^{5}$ D. Brunner, B. LaBombard, R. Ochoukov and D. Whyte, Rev. Sci. Instrum. 84, 033502 (2013).

${ }^{6}$ I. S. Nedzelskiy, C. Silva, H. Figueiredo, H. Fernandes and C. A. F. Varandas, Rev. Sci. Instrum. 77, (2006).

${ }^{7}$ M. Dreval, D. Rohraff, C. Xiao and A. Hirose, Rev. Sci. Instrum. 80, 103505 (2009).

${ }^{8}$ Y. L. Li, G. S. Xu, C. Xiao, H. Q. Wang, N. Yan, B. N. Wan, L. Chen, Y. L. Liu, H. Zhang, W. Zhang, L. Wang, G. H. Hu, R. Chen, J. C. Xu, Y. Ye and J. Li, Rev. Sci. Instrum. 87, 123503 (2016).

${ }^{9}$ H. Renner, J. Boscary, V. Erckmann, H. Greuner, H. Grote, J. Sapper, E. Speth, F. Wesner and M. Wanner, Nucl. Fusion 40, 1083 (2000).

${ }^{10}$ T. Sunn Pedersen, R. König, M. Jakubowski, M. Krychowiak, D. Gradic, C. Killer, H. Niemann, T. Szepesi, U. Wenzel, A. Ali, G. Anda, J. Baldzuhn, T. Barbui, C. Biedermann, B. D. Blackwell, H. S. Bosch, S. Bozhenkov, R. Brakel, S. Brezinsek, J. Cai, B. Cannas, J. W. Coenen, J. Cosfeld, A. Dinklage, T. Dittmar, P. Drewelow, P. Drews, D. Dunai, F. Effenberg, M. Endler, Y. Feng, J. Fellinger, O. Ford, H. Frerichs, G. Fuchert, Y. Gao, J. Geiger, A. Goriaev, K. Hammond, J. Harris, D. 
Hathiramani, M. Henkel, Y. O. Kazakov, A. Kirschner, A. Knieps, M. Kobayashi, G. Kocsis, P. Kornejew, T. Kremeyer, S. Lazerzon, A. LeViness, C. Li, Y. Li, Y. Liang, S. Liu, J. Lore, S. Masuzaki, V. Moncada, O. Neubauer, T. T. Ngo, J.

Oelmann, M. Otte, V. Perseo, F. Pisano, A. Puig Sitjes, M. Rack, M. Rasinski, J. Romazanov, L. Rudischhauser, G. Schlisio, J. C. Schmitt, O. Schmitz, B. Schweer, S. Sereda, M. Sleczka, Y. Suzuki, M. Vecsei, E. Wang, T. Wauters, S. Wiesen, V. Winters, G. A. Wurden, D. Zhang and S. Zoletnik, Nucl. Fusion 59, (2019).

${ }^{11}$ C. Killer, O. Grulke, P. Drews, Y. Gao, M. Jakubowski, A. Knieps, D. Nicolai, H. Niemann, A. P. Sitjes and G. Satheeswaran, Nucl. Fusion 59, (2019).

${ }^{12}$ D. Nicolai, V. Borsuk, P. Drews, O. Grulke, K. P. Hollfeld, T. Krings, Y. Liang, C. Linsmeier, O. Neubauer, G. Satheeswaran, B. Schweer and G. Offermanns, Fusion Eng. Des. 123, 960 (2017).

${ }^{13}$ G. Satheeswaran, K. P. Hollfeld, P. Drews, D. Nicolai, O. Neubauer, B. Schweer and O. Grulke, Fusion Eng. Des. 123, 699 (2017).

${ }^{14}$ Y. Liang, O. Neubauer, R. König, M. Krychowiak, B. Schweer, P. Denner, M. Rack, D. Reiter, Y. Feng, A. KrämerFlecken, P. Drews, F. Hasenbeck, S. Liu, Y. Gao, E. H. Wang, Y. Wei, M. Dostal, L. Li, N. Wang, J. Geiger, Y. Suzuki, S. Sereda, P. Börner, A. C. Weger, W. Biel, S. Brezinsek, A. Charl, G. Czymek, D. Höschen, F. Effenberg, O. Grulke, D. Nicolai, H. T. Lambertz, O. Marchuk, O. Schmitz, K. P. Hollfeld, M. Knaup, G. Offermanns, G. Satheeswaran, A. Terra, J. Thomas, T. S. Pederson, U. Samm and C. Linsmeier, Nucl. Fusion 57, (2017).

${ }^{15}$ P. Drews, C. Killer, A. Knieps, M. Henkel, S. Brezinsek, T. Dittmar, J. Cai, A. Card, O. Grulke, D. Höschen, M. Jakubowski, R. König, A. Krämer-Flecken, Y. Liang, Y. Li, C. Linsmeier, S. Liu, D. Nicolai, O. Neubauer, G. Satheeswaran, N. Sandri, B. Schweer and T. Schröder, Fusion Eng. Des., (2019).

${ }^{16}$ J. Cai, Y. Liang, C. Killer, S. Liu, A. Hiller, A. Knieps, B. Schweer, D. Höschen, D. Nicolai, G. Offermanns, G. Satheeswaran, M. Henkel, K. Hollfeld, O. Grulke, P. Drews, T. Krings and Y. Li, Rev. Sci. Instrum. 90, 033502 (2019). ${ }^{17}$ M. Henkel, D. Höschen, Y. Liang, Y. Li, S. C. Liu, D. Nicolai, N. Sandri, G. Satheeswaran, N. Yan and H. X. Zhang, Plasma Sci. Technol. 20, 054001 (2018).

${ }^{18}$ S. C. Liu, Y. Liang, P. Drews, C. Killer, A. Knieps, G. S. Xu, H. Q. Wang, N. Yan, X. Han, D. Höschen, A. KrämerFlecken, D. Nicolai, G. Satheeswaran, K. Hammond, J. Q. Cai, A. Charl, J. Cosfeld, G. Fuchert, Y. Gao, J. Geiger, O. Grulke, M. Henkel, M. Hirsch, U. Hoefel, R. König, Y. Li, O. Neubauer, E. Pasch, K. Rahbarnia, M. Rack, N. Sandri, S. Sereda, B. Schweer, E. H. Wang, S. Xu and X. Gao, Nucl. Fusion 59, (2019). 